\title{
Liquid-liquid phase coexistence for prebiotic compartmentalization
}

\section{CHRISTINE D KEATING}

Penn State University

Presenting Author: cmd8@psu.edu

Liquid-liquid phase separation can produce membraneless compartments called coacervates provide distinct microenvironments, accumulate solutes, and facilitate reactions. Due to their ease of formation, ability to accumulate molecules such as RNA from dilute solutions and to enhance ribozyme catalysis, coacervate droplets have been considered as prebiotic compartments in the origins of life. This presentation will focus on the physical chemistry of simple model systems composed of phase-separating aqueous polyelectrolyte solutions, with an eye towards their feasibility as prebiotic compartments. It will explore the effect of environmental wet/dry cycling on oligoRNA compartmentalization by coacervates, with particular emphasis on how the observed changes can be understood through consideration of the phase diagram. It will also evaluate the impact of lower, more prebiotically-relevant, polyion multivalency on the functional performance of coacervates as compartments. Surprisingly, we find that compartments formed from lower-molecular weight polyions can outperform their higher-molecular weight analogues for some compartmentalization functions. 